\section{An efficient method for purification of PCR products for sequencing}

\author{
Hao Ma and Stephen DiFazio \\ Department of Biology, West Virginia University, Morgantown, WV, USA \\ BioTechniques 44:921-923 (June 2008) \\ doi 10.2144/000112809
}

\begin{abstract}
A high-throughput DNA sequencing method that generated high quality data was developed. A frame fashioned from a standard agarose gel combined with $0.1 \%-0.2 \%$ low-melting point (LMP) agarose gel was used to isolate the PCR product of interest. Collected PCR products were centrifuged without any reagents and the supernatants were directly used for a sequencing reaction. This method is simple and labor efficient, provides high quality sequences at a low cost, and bypasses problems with impure PCR products. This technique has been used for single nucleotide polymorphism (SNP) discovery in Populus angustifolia trees.
\end{abstract}

Quality DNA sequencing is one of the most important tools in molecular biology research. Because the genomes of more than 300 organisms have been sequenced and another 750 are in process (1), there is increased demand for high-throughput and low-cost methods for resequencing genomes in order to identify genetic variants and make inferences about gene function (2). Because the Sanger sequencing method provides relatively long read lengths and low error rates $(3,4)$, direct sequencing of PCR products with the Sanger-based method is one of the most widely used procedures for resequencing (4-7).

PCR products can be either cloned or directly used for Sanger sequencing. The former procedure involves cloning, transformation, bacterial incubation, and DNA isolation steps to prepare template DNA for sequencing. Direct DNA sequencing of PCR products is a much more efficient approach. If a single fragment is amplified, PCR products can be directly sequenced after removal of PCR primers and/or inactivation of dNTPs with phosphatase (8). However, PCR products are often comprised of a mixture of nonspecific products in addition to the target product. In this case, the band of interest must be isolated, usually from an agarose gel, followed by costly and time-consuming clean-up procedures prior to sequencing $(9,10)$.
We have developed a procedure using a combination of standard agarose and low-melting point (LMP) agarose to purify and recover PCR products, which can then be sequenced directly with little additional purification. The agarose gel is initially prepared using a standard gel mold [Model A2 $(28 \mathrm{~cm}$ $\mathrm{W} \times 37.5 \mathrm{~cm} \mathrm{~L} \times 11 \mathrm{~cm} \mathrm{H})$ or B2 (18 $\mathrm{cm} \mathrm{W} \times 24.5 \mathrm{~cm} \mathrm{~L} \times 9.5 \mathrm{~cm} \mathrm{H}$ ); Owl Separation Systems, Portsmouth, NH, USA], after which a rectangle is cut from the interior portion, where the LMP agarose is subsequently poured (Figure 1). Size separation takes place primarily in the standard agarose portion of the gel, which can be varied in length and concentration to increase the resolution of the bands. The products then enter the LMP portion of the gel, from which they can be easily pipeted and sequenced directly. We initially tested five different concentrations of LMP agarose gel (i.e., $0.1 \%, 0.2 \%$. $0.4 \%, 0.6 \%$, and $0.8 \%$ ). Concentrations $>0.2 \%$ LMP agarose interfered with pipeting and prevented the formation of firm pellets in 96well plates, often resulting in loss of the pellet upon inversion of the plate. Templates pipeted from $0.2 \%$ and $0.1 \%$ LMP gel consistently generated strong sequencing signals.

Previous studies have shown that agarose gel concentrations $>0.2 \%$ inhibit sequencing reactions (5). Our method ensured that the agarose concentration was $<0.2 \%$ in the sequencing reaction, since we began with a $0.1-0.2 \%$ LMP gel, and the LMP agarose was then further diluted in the sequencing reaction. Consequently, the remaining agarose should not affect the sequencing reaction. On the other hand, the very low concentration of agarose in the sequencing reaction might even improve the sequence length and quality, as has been shown previously (11).

We tested our method using eight loci from the Populus angustifolia genome using 30 to 32 trees collected from wild populations in Utah (12).

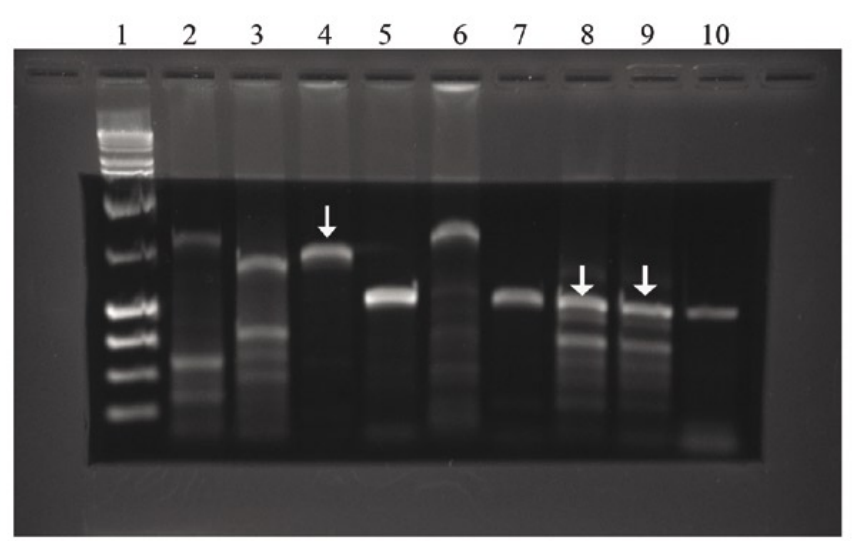

Figure 1. PCR reactions electrophoresed in a $2.0 \%$ agarose gel frame with an embedded LMP agarose gel reservoir. Composition of each lane (product/template): 1, $1 \mathrm{~Kb}$ DNA Ladder; 2, HSP1_0834_2477/Sample 15; 3, HSP1_2121_3424/Sample 15; 4, Brun1_2002_3355/Sample 15; 5, Brun2_2175_3188/Sample 15; 6, Brun24470_5959/Sample 15; 7, PTLF_1094_2078/Sample 15; 8, PTLF_2059_3046/Sample 15; 9, PTLF_2059_3046/Sample 19; 10, TFL_2019_2976/Sample 15. White arrows indicate PCR products for which sequences are shown in Figure 2. 


\section{Benchmarks}

Primer pairs that gave single or multiple PCR products were tested using the BigDye Terminator v3.1 Cycle Sequencing kit on a 3130x1 DNA Genetic Analyzer (Applied Biosystems, Foster City, CA, USA). With this method, the pass rate $(>100$ bp with Phred $>$ Q20) ranged from $92 \%$ to $100 \%$. The average read length ranged from 549 to 744 Q20 bases using a $50 \mathrm{~cm}$ capillary on the ABI 3130xl DNA Genetic Analyzer.
The average read length ranged from 483 to 526 Q20 bases with a $35 \mathrm{~cm}$ capillary (Table 1). Direct sequencing of the PCR products generated strong signals and low background intensities, such that the SNPs were readily identified in the sequence profiles (Figure 2). The quality of the sequence is demonstrated by high Phred quality scores, which were $>47$ on average (Table 1 ). These high quality sequences were
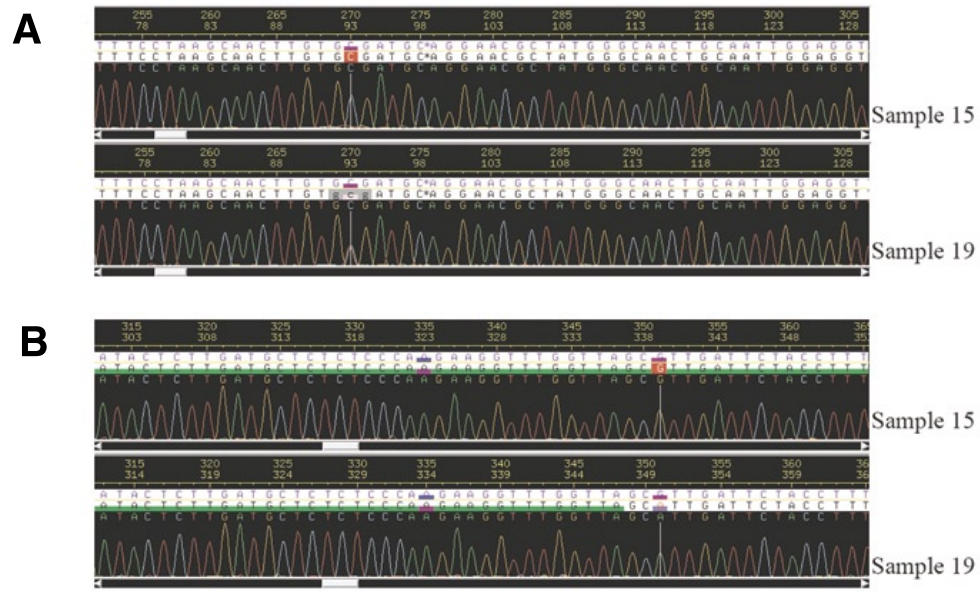

Figure 2. Sequencing results obtained from single and multiple PCR products. Equally high quality sequences were obtained from products with clear single bands as from products with multiple bands. Images were captured from Consed and scored with PolyPhred $(14,15)$. The SNPs are clearly scorable from these sequences (indicated by boxes color-coded by PolyPhred). (A) Product Brun1_2002_3355 with single PCR product, forward primer, samples 15 and 19. (B) Product PTLF_2059_3046 with multiple PCR products, forward primer, samples 15 and 19.

obtained even with a 1:8 dilution of the recommended quantity of BigDye Terminator v3.1 kit and a total sequencing reaction volume of only 5 $\mu \mathrm{L}$. Finally, a high quality sequence was obtained even when multiple products were amplified by a given primer set (Table 1; Figure 2).

This method provides many advantages over traditional procedures for direct sequencing of PCR products. First, pipeting the PCR product from the LMP gel to a new tube is simpler and easier than excising the gel slice. Second, the procedure was successful even for impure PCR products with nonspecific amplification. The concentration of standard agarose and the position of the LMP agarose can be altered according to the number of bands and proximity of nonspecific bands to the target band because enhanced separation can be achieved with longer runs through standard agarose. The disadvantages of isolating DNA from LMP gels, including poor resolution of closely migrating bands, can be overcome by this method (9). Third, the method is highly scalable, and could be automated using robotics, particularly for unique PCR products. Finally, this method greatly decreases the cost of direct sequencing. The $1.0 \%-2.0 \%$ agarose gel frame is reusable and the concentration of LMP agarose is $0.1 \%-0.2 \%$, so little agarose

Table 1. Summary of Sequencing Quality for Selected Populus trichocarpa Loci Resequenced in Populus angustifolia

\begin{tabular}{|c|c|c|c|c|}
\hline Product & Number of Samplesa & Pass Rateb & Average Read Lengthc & Average Phred Qualityd \\
\hline HSP1_0834_2477 & 61 & 1.00 & $626.15 \pm 131.01$ & $52.73 \pm 8.60$ \\
\hline Brun1_2002_3355 & 64 & 0.92 & $515.21 \pm 144.33$ & $50.40 \pm 7.19$ \\
\hline Brun2_4470_5959 & 62 & 0.97 & $548.75 \pm 233.15$ & $51.87 \pm 7.58$ \\
\hline PTLF_1094_2078e & 64 & 1.00 & $483.13 \pm 108.61$ & $50.04 \pm 10.06$ \\
\hline PTLF_2059_3046 & 64 & 0.91 & $599.07 \pm 218.00$ & $48.65 \pm 13.47$ \\
\hline TFL_2019_2976e & 64 & 0.98 & $526.11 \pm 25.36$ & $57.51 \pm 5.63$ \\
\hline
\end{tabular}


Mutation Discovery, Genotyping and Gene Scanning

is consumed. In fact, we calculate that the total cost of LMP agarose is approximately $\$ 0.02$ per sample, compared with up to $\$ 1.80$ per sample for commercially available gel purification kits such as the QIAquick Gel Extraction kit (Qiagen, Germantown, MD, USA). Therefore, this approach is one of the most efficient methods currently available for direct sequencing, and should be a boon for labs on a tight sequencing budget.

\section{ACKNOWLEDGEMENTS}

We thank Gancho Slavov for reviewing the manuscript. We also thank David Ray, Danielle Ellis, Brahma Reddy Induri, and Dhiraj Naik for help with laboratory work. This work was supported by a grant from the West Virginia University Research Corporation and National Science Foundation (no. FIBR-0425908).

\section{COMPETING INTERESTS STATEMENT}

The authors declare no competing interests.

\section{REFERENCES}

1. Bonetta, L. 2006. Genome sequencing in the fast lane. Nat. Methods 3:141-147.

2. Ruparel, H., L. Bi, X. Bai, D.H. Kim, N.J. Turro, and J. Ju. 2005. Design and synthesis of a 3'-O-allyl photocleavable fluorescent nucleotide as a reversible terminator for DNA sequencing by synthesis. Proc. Natl. Acad. Sci. USA 102:5932-5937.

3. Blazej, R.G., P. Kumaresan, and R.A. Methies. 2006. Microfabricated bioprocessor for integrated nanoliter-scale Sanger DNA sequencing. Proc. Natl. Acad. Sci. USA 103:7240-7245.

4. Yao, F., R. Zhang, Z. Zhu, K. Xia, and C. Liu. 2006. MutScreener: primer design tool for PCR-direct sequencing. Nucleic Acids Res. 34:W660-W664.

5. Yamaguchi, Y., S. Nimbari, T. Ookawara, K. Oishi, H. Eguchi, and K. Suzuki. 2002. Inhibitory effects of agarose gel and LB medium on DNA sequencing. BioTechniques 33:282-284.

6. Witonsky, J. 2007. BioMarket trends: DNA sequencing. Genet. Eng. \& Biotech. News 27:1 [Epub].

7. Platt, A.R., R.W. Woodhall, and A.F. George, Jr. 2007. Improved DNA sequencing quality and efficiency using an optimized fast cycle sequencing protocol. BioTechniques 43:58-62.

8. Le Hellard, S., S.J. Ballereau, P.M. Visscher, H.S. Torrance, J. Pinson, S.W. Morris, M.L. Thomson, C.A. Semple, et al. 2002. SNP genotyping on pooled DNA: comparison of genotyping technologies and a semi automated method for data storage and analysis. Nucleic Acids Res. 30:e74.

9. Seelan, R.S. and L.I. Grossman. 1991. A rapid protocol to isolate DNA fragments from low melting temperature agarose. BioTechniques 10:186-187.

10. Leonard, J.T., M.B. Grace, G.S. Buzard, M.J. Mullen, and C.B. Barbagallo. 1998. Preparation of PCR products for DNA sequencing. BioTechniques 24:314-317.

11. Vatcher, G., D. Smailus, M. Krzywinski, R. Guin, J. Stott, M. Tsai, S. Chan, P. Pandoh, et al. 2002. Resuspension of DNA sequencing reaction products in agarose increases sequence quality on an automated sequencer. BioTechniques 33:532-538

12. Martinsen, G.D., T.G. Whitham, R.J. Turek, and P. Keim. 2001. Hybrid populations selectively filter gene introgression between species. Evolution Int. J. Org. Evolution 55:1325-1335.

13. Ewing, B., L. Hillier, M.C. Wendle, and P. Green. 1998. Base-calling of automated sequencer traces using Phred. I. Accuracy assessment. Genome Res. 8:175-185.

14. Gordon, D., C. Abajian, and P. Green. 1998. Consed: A graphical tool for sequence finishing. Genome Res. 8:195-202.

15. Nickerson, D.A., V.O. Tobe, and S.L. Taylor. 1997. PolyPhred: Automating the detection and genotyping of single nucleotide substitutions using fluorescence-based resequencing. Nucleic Acids Res. 25:2745-2751.

Received 22 October, 2007; accepted 29 January 2008.

Address correspondence to Hao Ma, Department of Biology, West Virginia University, 53 Campus Drive, Morgantown, WV 26506, USA. e-mail: hao.ma@mail. wvu.edu

To purchase reprints of this article, contact: Reprints@BioTechniques.com

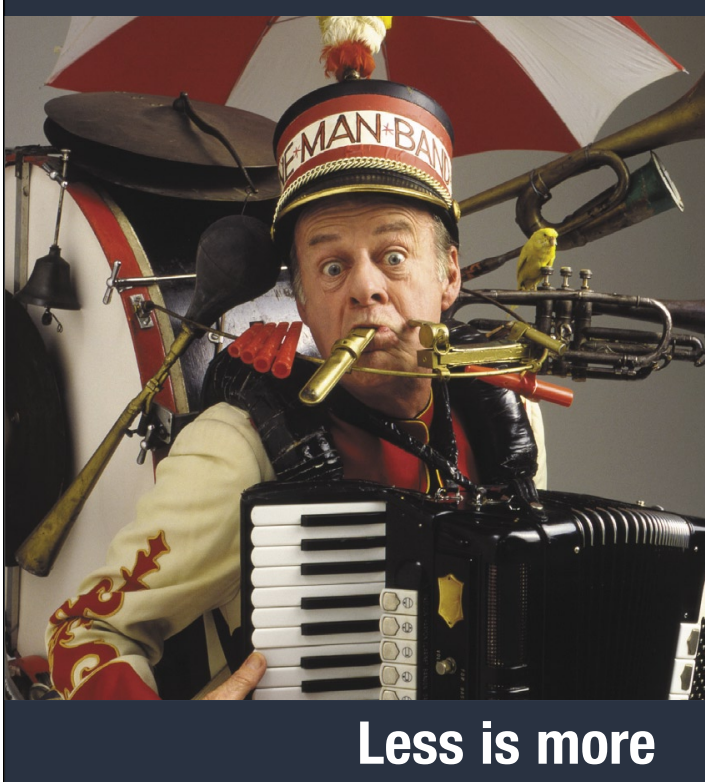

Don't be fooled by other instruments that are designed for real-time PCR but claim to perform "HRM". Only with the LightScanner do you get a system built by the same people who invented the technology and developed the chemistry.

The LightScanner ${ }^{\circledR}$ System, is the premier instrument for mutation discovery and genotyping using Hi-Res Melting ${ }^{\mathrm{TM}}$ technology.

\section{Leading the Industry in Hi-Res Melting Curve Analysis}

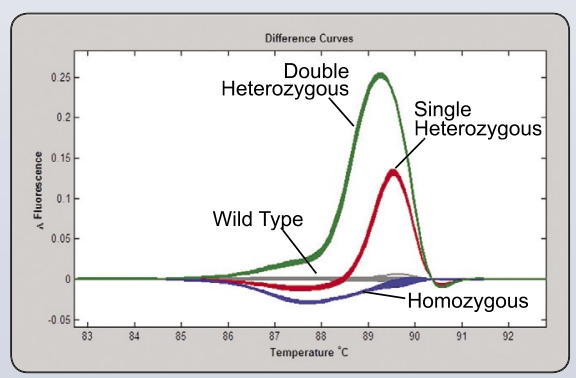

Mutation Scanning Data Analysis: Four distinct groups can be identified by the difference compared to the wild type sample.

"Designed for Hi-Res Melting" lets you know that you have chosen the RIGHT tool for the job.

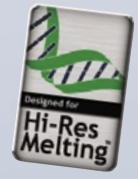

